\title{
A limited CpG-containing oligodeoxynucleotide therapy regimen induces sustained suppression of allergic airway inflammation in mice
}

\author{
John D Campbell, ${ }^{1}$ Sariah A Kell, ${ }^{1}$ Heather M Kozy, ${ }^{1}$ Jeremy A Lum, ${ }^{1}$ \\ Rosemary Sweetwood, ${ }^{1}$ Mabel Chu ${ }_{1}^{1}$ Cameron R Cunningham, ${ }^{1}$ Hugh Salamon, ${ }^{2}$ \\ Clare M Lloyd, ${ }^{3}$ Robert L Coffman, ${ }^{1}$ Edith M Hessel ${ }^{1}$
}

\begin{abstract}
- Additional material is published online only. To view please visit the journal online (http://dx.doi.org/10.1136/ thoraxjnl-2013-204605).

${ }^{1}$ Dynavax Technologies, Berkeley, California, USA ${ }^{2}$ Knowledge Synthesis, Berkeley, California, USA ${ }^{3}$ Leukocyte Biology Section, National Heart and Lung Institute, Imperial College London, London, UK
\end{abstract}

\section{Correspondence to} Dr John D Campbell, Dynavax Technologies, 2929 7th St, Ste 100, Berkeley, CA 94710, USA: dcampbell@dynavax.com

JDC and SAK shared first authorship and contributed equally.

Received 3 October 2013 Revised 24 December 2013 Accepted 9 January 2014 Published Online First 24 January 2014

\begin{abstract}
Background CpG-containing oligodeoxynucleotides (CpG-ODNs) are potent inhibitors of T helper 2 mediated allergic airway disease in sensitised mice challenged with allergen. A single treatment has transient effects but a limited series of treatments has potential to achieve clinically meaningful sustained inhibition of allergic airway disease.
\end{abstract}

Objective To optimise the treatment regimen for sustained efficacy and to determine the mechanisms of action in mice of an inhaled form of CpG-ODN being developed for human asthma treatment.

Methods We set up a chronic allergic-asthma model using ragweed-sensitised mice exposed weekly to intranasal ragweed. Using this model, the effects of a limited series of weekly intranasal 1018 ISS (CpG-ODN; B-class) treatments were evaluated during treatment and for several weeks after treatments had stopped but weekly allergen exposures continued. Treatment efficacy was evaluated by measuring effects on lung $T$ helper 2 cytokines and eosinophilia, and lung dendritic cell function and T-cell responses.

Results Twelve intranasal 1018 ISS treatments induced significant suppression of bronchoalveolar lavage eosinophilia and interleukin 4, 5 and 13 levels. This suppression of allergic $T$ helper 2 parameters was maintained through 13 weekly ragweed exposures administered after treatment cessation. Subsequent experiments demonstrated that at least five treatments were required for lasting suppression. Although CpGODN induced moderate T helper 1 responses, suppression of allergic airway disease did not require interferon $\gamma$ but was associated with induction of a regulatory T-cell response.

Conclusions A short series of CpG-ODN treatments results in sustained suppression of allergic lung inflammation induced by a clinically relevant allergen.

\section{INTRODUCTION}

Current treatments for allergic asthma provide temporary relief but do not address the causes underCrossMark lying allergic disease. ${ }^{1}$ While allergen-specific immunotherapy provides long-term relief for allergic rhinitis, it is less effective for asthma. ${ }^{2-4}$ Thus, the need to develop treatment alternatives for asthma that have long-lasting, disease-modifying effects is apparent. ${ }^{5}$

\section{Key messages}

What is the key question?

- Can CpG-containing oligodeoxynucleotide (CpG-ODN) treatments induce lasting suppression of allergic airway disease?

What is the bottom line?

- A limited weekly series of intranasal CpG-ODN treatments induced a $\mathrm{T}$ helper 2 to $\mathrm{T}$ helper 1 and regulatory T-cell shift in the airway and long-lasting suppression of allergic airways disease despite ongoing allergen exposures.

\section{Why read on?}

- Delivery of CpG-ODN directly to the lungs represents a potential disease-modifying therapy for extended remission from allergic asthma.

The potential of $\mathrm{CpG}$-containing oligodeoxynucleotides (CpG-ODNs) as an immunemodulating therapy for allergic asthma has been demonstrated in animal models. CpG-ODNs stimulate innate immune responses through tolllike receptor 9 (TLR9) expressed principally by plasmacytoid dendritic cells (pDCs) and B cells in humans. ${ }^{6}$ In allergen challenge models, prophylactic or therapeutic CpG-ODN administration inhibited airway hyperresponsiveness, mucus secretion, eosinophilia and T helper 2 (Th2) cytokines. $^{7-10}$ Three to six-month treatment models in mice and monkeys demonstrate inhibition of lung inflammation and remodelling with continued CpG-ODN dosing. ${ }^{11-14}$ However, enduring suppression of Th2 responses in the presence of frequent allergen exposures long after completion of a limited CpG-ODN treatment course, a model more relevant to clinical application, has not been demonstrated in animal models of allergic asthma.

Evidence that brief treatment with $\mathrm{CpG}-\mathrm{ODN}$ could produce long-term suppression of allergic disease comes from clinical studies using the CpG-ODN 1018 ISS conjugated to the ragweed (RW) allergen $A m b$ a 1 . Studies showed that six 
doses lowered nasal and systemic Th2 responses with reduction in allergy symptoms for two RW seasons after treatment. ${ }^{15-17}$

CpG-ODN therapy thus has potential for disease-modifying effects. The optimum strategy for treating allergic asthma, usually triggered by multiple allergens, is application of nonconjugated $\mathrm{CpG}-\mathrm{ODN}$ directly to the respiratory tract, the site of allergen exposure. However, treatment optimisation requires an understanding of the regimens and mechanisms needed to produce long-term allergic disease suppression. Thus, we developed a chronic mouse model focused on therapeutic delivery of free 1018 ISS directly to the airways via intranasal administration. We demonstrate that a short series of weekly treatments induces months-long inhibition of RW-induced lung Th2 responses. We assessed the contributions of dendritic cell function and T-cell responses to maintenance of this CpG-ODN-induced disease modification.

\section{MATERIALS AND METHODS}

\section{Chronic allergic model and CpG-ODN therapy}

Female BALB/c mice (6-8 weeks old; Charles River) were sensitised intraperitoneally with $15 \mu \mathrm{g}$ RW (Ambrosia artemissifolia;
Greer Laboratories) in alum on days 0 and 7. Starting day 14, mice received, under isofluorane anaesthesia, weekly intranasal low-dose RW $(0.5 \mu \mathrm{g})$ exposures for 1-26 weeks, were rested for 2 weeks and given a final high dose $(5 \mu \mathrm{g}) \mathrm{RW}$ challenge $24 \mathrm{~h}$ before sacrifice. $20 \mu \mathrm{g} \quad 1018$ ISS (B-class; 5'-TGACTGTGAACGTTCGAGATGA), synthesised as previously described, ${ }^{18}$ was co-administered with RW to indicated groups (Tx protocol 1; figure 1A). In certain experiments, RW +1018 ISS administration was limited to a set number of weeks, after which mice received only weekly RW exposures (Tx proto$\operatorname{col} 2$; figure 2A) before rest and final RW challenge.

Tissue harvest and cell isolation

Serum was isolated using separation tubes (BD Biosciences). Bronchoalveolar lavage fluid (BALF) was processed and cytospins prepared for differential counting as previously described. ${ }^{19}$ Single-cell suspensions from treatment group pooled lung tissue, spleen or lymph nodes were also prepared. Peripheral blood mononuclear cells (PBMCs) were isolated from heparinised mouse blood using Lympholyte (CedarLane) according to manufacturer's instructions.

\section{A $\mathrm{RW}+1018$ ISS Tx Protocol 1}
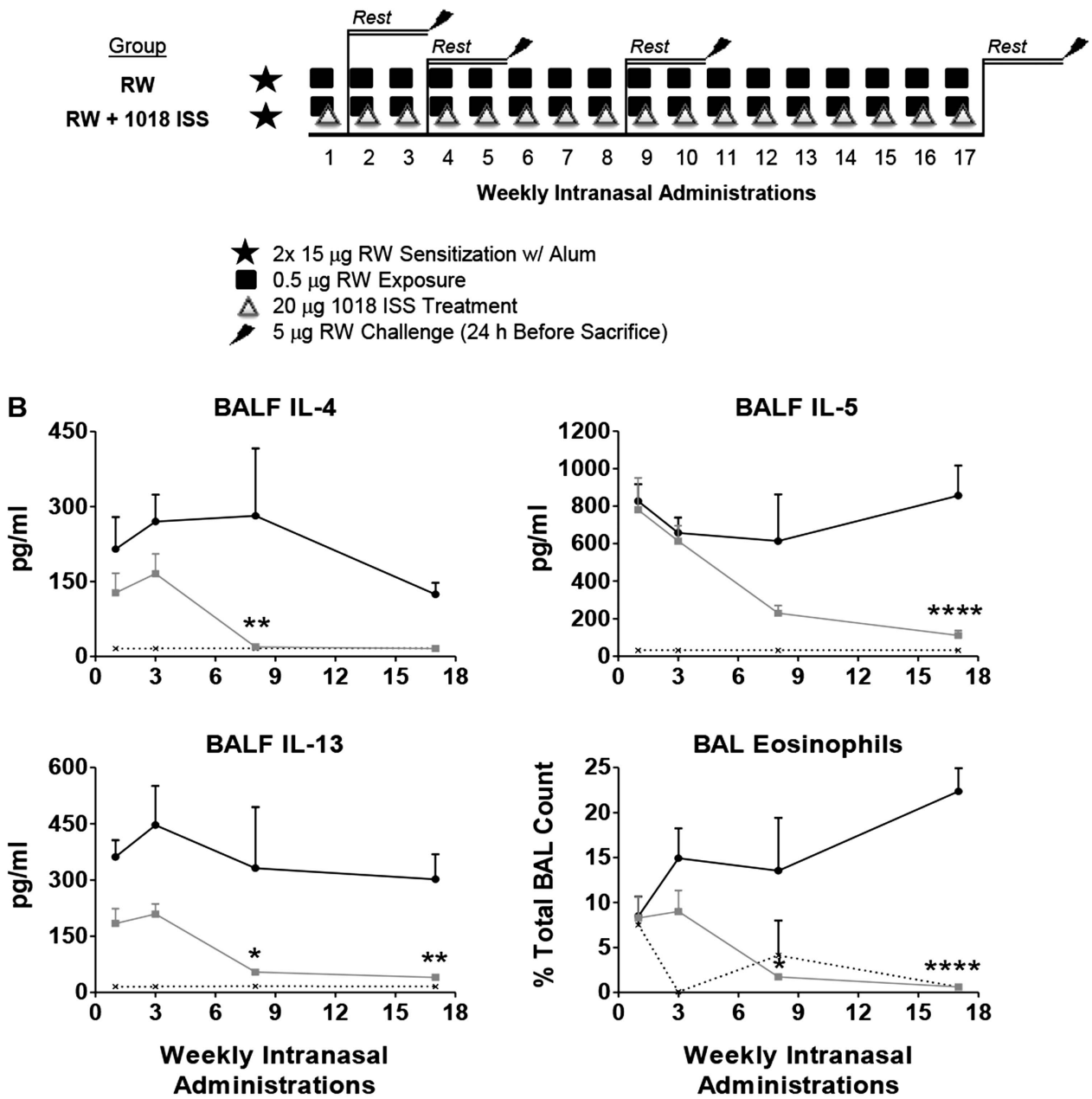

$$
\text { **. Saline } \rightarrow \mathrm{RW} \rightarrow \mathrm{RW}+1018 \text { ISS Tx Protocol } 1
$$

Figure 11018 ISS treatments progressively suppress airway T helper 2 responses. (A) Tx protocol 1 schematic: mice received 1, 3, 8 or 17 weekly intranasal saline, $0.5 \mu \mathrm{g}$ ragweed (RW), or RW+20 $\mu \mathrm{g} 1018$ ISS treatments, were rested 2 weeks, and then given a final $5.0 \mu \mathrm{g}$ RW challenge $24 \mathrm{~h}$ before sacrifice. (B) Bronchoalveolar lavage fluid (BALF) cytokines levels and eosinophil numbers (mean $\pm S E M, 5$ mice/group). ${ }^{*} p<0.05,{ }^{* *} p<0.01$, ${ }^{* * *} \mathrm{p}<0.0001$ compared with the RW only exposed group. Data are representative of four independent experiments. IL, interleukin. 
A $\underline{\text { RW }+1018 \text { ISS Tx Protocol } 2}$

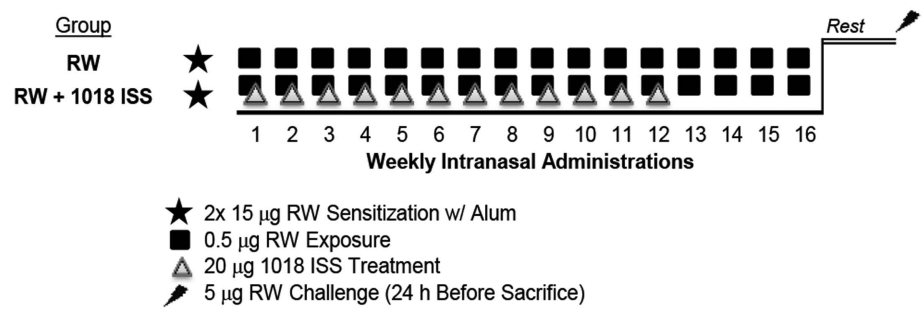

B Tx Protocol 1 versus Tx Protocol 2 (+4 weekly RW exposures post 1018 ISS treatments)
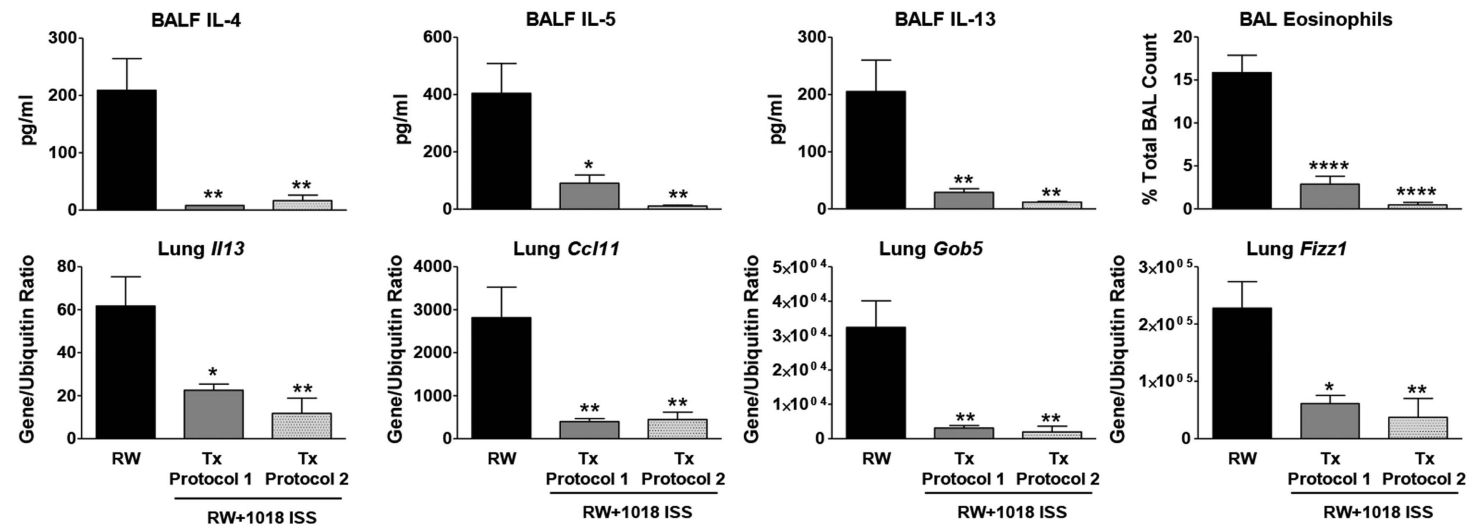

C Ix Protocol 1 versus Tx Protocol 2 (+13 weekly RW Exposures post 1018 ISS treatments)
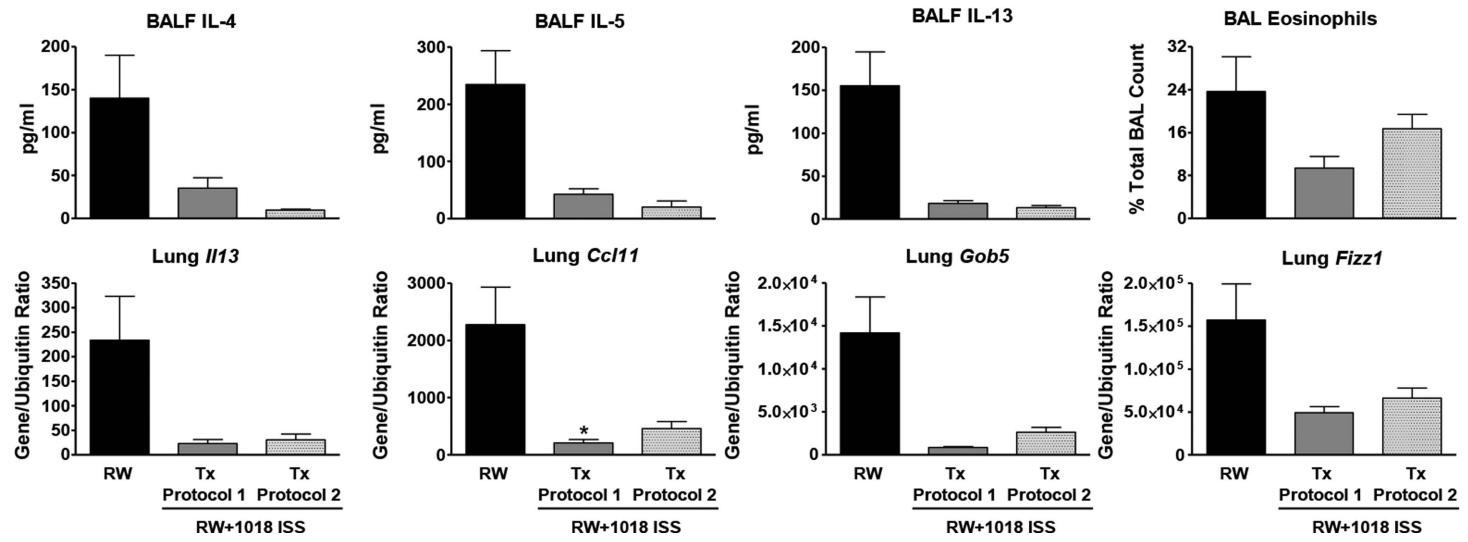

Figure 2 A limited series of 1018 ISS treatments induces lasting suppression of airway T helper 2 responses. (A) Tx protocol 2 schematic: groups were similar to Tx protocol 1 except that 1018 ISS was given for 12 weeks, followed by either 4 (B) or 13 (C) weekly ragweed (RW) only exposures followed by rest and final RW challenge. Bronchoalveolar lavage fluid (BALF) cytokines, eosinophils and lung gene expression are shown (mean \pm SEM, 5 mice/group). ${ }^{*} p<0.05,{ }^{* *} p<0.01$ and ${ }^{* * * *} p<0.0001$ compared with the RW only exposed group. Data are representative of four independent experiments. IL, interleukin.

\section{ELISA}

BALF cytokines and serum antibodies were quantified using matched antibody pairs (BD Biosciences) or ELISA kits (R\&D Systems or Alpha Diagnostics International) according to manufacturer's instructions. Lower limits of detection were 8, 16, 8, 8 and $13 \mathrm{pg} / \mathrm{mL}$ for interleukin (IL)-4, IL-5, IL-13, IL-10 and interferon $\gamma(\mathrm{IFN} \gamma)$, and 250 and $100000 \mathrm{ng} / \mathrm{mL}$ for $\operatorname{IgE}$ and IgG2a, respectively.

\section{Gene expression analysis}

Real-time PCR of lung tissue RNA was performed as previously described. ${ }^{18} 19$ Data are normalised as gene/ubiquitin ratio. Microarray analysis was performed by Expression Analysis, Inc. (Durham, North Carolina, USA).
Bone marrow-derived dendritic cell generation and adoptive transfer

Bone marrow was harvested from naïve $\mathrm{BALB} / \mathrm{c}$ mouse femurs and marrow-derived dendritic cells (BMDCs) were generated as per Lutz et al's protocol. ${ }^{20}$ After 9 days of maturation, BMDCs were RW pulsed $(100 \mu \mathrm{g} / \mathrm{mL})$ overnight and washed prior to adoptive transfer. BMDCs were typically $80-85 \% \mathrm{I}_{-} \mathrm{A}^{\mathrm{d}+}$ and CD $11 \mathrm{c}^{+}$. Mice received $2 \times 10^{6}$ RW-pulsed or non-pulsed BMDCs intratracheally as a final challenge $24 \mathrm{~h}$ before sacrifice, following Tx protocol 2. Control mice received RW $(5 \mu \mathrm{g})$ or saline intratracheally.

T-cell analysis

Enriched lung $\mathrm{T}$ cells were stained with surface markers. Intracellular cytokine responses of group-pooled lung $\mathrm{T}$ cells 
were measured using de novo CD154 expression to identify antigen-specific $\mathrm{T}$ cells ex vivo, ${ }^{21}$ unless otherwise specified. Fixed cells were analysed on a FACSCaliber or LSRII flow cytometer (BD Biosciences). Ex vivo activity of lung CD25 ${ }^{+} \mathrm{T}_{\text {Reg }}$ cells was assessed by co-incubation with $\mathrm{CD} 25^{-}$cells and measurement of anti-CD3-induced proliferation by thymidine incorporation.

\section{Statistical analysis}

Individual sample data are expressed as mean \pm SEM. All study groups were tested for equal variance and compared with the respective RW-exposed group using one-way or two-way analysis of variance, the Mann-Whitney $U$ test or $t$ test. A p value of $<0.05$ was regarded as statistically significant.

Additional details on the methods used are presented in the online supplementary data file.

\section{RESULTS}

\section{A series of 1018 ISS treatments induces long-lasting} suppression of allergic responses in chronically RW-exposed mice

To test whether ongoing CpG-ODN treatments could achieve lasting inhibition of lung Th2 responses, we developed an allergic asthma model with a treatment and allergen exposure pattern intended to mimic clinical delivery of inhaled CpG-ODN to patients. After sensitisation to RW in alum, mice were exposed weekly to low-dose intranasal $(0.5 \mu \mathrm{g})$ RW. Treated mice received $20 \mu \mathrm{g} 1018$ ISS intranasally with weekly RW exposures (Tx protocol 1, figure 1A). Treatment protocol 1 represents concurrent weekly allergen exposures and 1018 ISS treatments. Groups of mice were sacrificed after 1, 3, 8 and 17 weeks to determine how many treatments were required to suppress inflammation in the presence of ongoing allergen challenges. To assess treatment effects without the confounding effects of the direct response to 1018 ISS, mice were rested for 2 weeks before a final challenge with a high dose $(5 \mu \mathrm{g})$ of RW. Control mice were RW sensitised, but exposed to saline thereafter.

Chronic RW exposures induced Th2-polarised lung inflammation, with large increases in IL-4, IL-5 and IL-13 protein, and BAL eosinophilia (figure 1B), which continued through 17 weekly allergen challenges. Th2 parameters were also elevated in mice given 1-3 weekly treatments with 1018 ISS in conjunction with weekly RW exposures (Tx protocol 1), indicating that allergic airway inflammation was initially evident in these mice. By 8 weeks of RW +1018 ISS treatments there was a sharp reduction to background in all markers of Th2 activity which continued through 17 weeks, the final time point assessed (figure 1B). Eight weeks of 1018 ISS treatments were also efficacious when treatment initiation started after mice received 3 weeks of RW only intranasal exposures (modified Tx protocol 1 ; see online supplementary figure $\mathrm{S} 1$ ), further demonstrating that a series of 1018 ISS treatments can reverse established allergic airway inflammation.

To determine whether a limited course of CpG-ODN treatment had durable effects in chronically allergen-exposed mice, mouse groups received weekly allergen exposures after completion of 1018 ISS therapy (Tx protocol 2, figure 2A). Treatment protocol 2 represents a limited course of weekly allergen and 1018 ISS exposures, followed by weekly RW exposures alone. Strikingly, allergic responses were equally inhibited by 12 weekly RW +1018 ISS treatments followed by 4 weekly RW exposures (Tx protocol 2) vs 16 weekly RW+1018 ISS treatments (Tx protocol 1). BALF Th2 cytokines, eosinophil levels and lung Th2-associated genes Il13, Ccl11, Gob5 and Fizz1 were suppressed equally with either protocol (figure 2B). Tx protocol 2 was also highly effective when 13 weekly RW exposures were administered after 12 weekly RW+1018 ISS treatments (figure 2C). Thus, a limited series of CpG-ODN treatments inhibited allergic lung inflammation for over 3 months after treatment in the presence of continued weekly allergen exposures.

To determine the minimum number of treatments required for disease modification, Tx protocol 2 was varied to include groups receiving $1,3,5$ or 8 weekly RW +1018 ISS treatments, followed by 4 weekly RW exposures each. At least 5 weekly 1018 ISS treatments were required to achieve this significant, lasting reduction of Th2-mediated responses to continued allergen exposure (figure 3 ).

\section{ISS treatments induce a shift from a RW-specific Th2} to Th1 response

To determine whether CpG-ODN treatments induced a Th1 shift in lungs of RW allergen-exposed mice, we measured lung
Figure 3 Five weekly 1018 ISS treatments are required for durable suppression of allergen-induced $T$ helper 2 responses in the airways. Mice were administered Tx protocol 2 with $1,3,5$ or 8 weekly ragweed (RW) +1018 ISS treatments followed by 4 weekly RW exposures compared with mice given only weekly RW exposures. All mice were lastly rested 2 weeks and administered a final RW challenge. Bronchoalveolar lavage fluid (BALF) cytokines and eosinophils are depicted (mean \pm SEM, 5 mice/group). ${ }^{*} \mathrm{p}<0.01$, $* * * p<0.001$ compared with the RW only exposed group. Data are representative of two independent experiments. IL, interleukin.
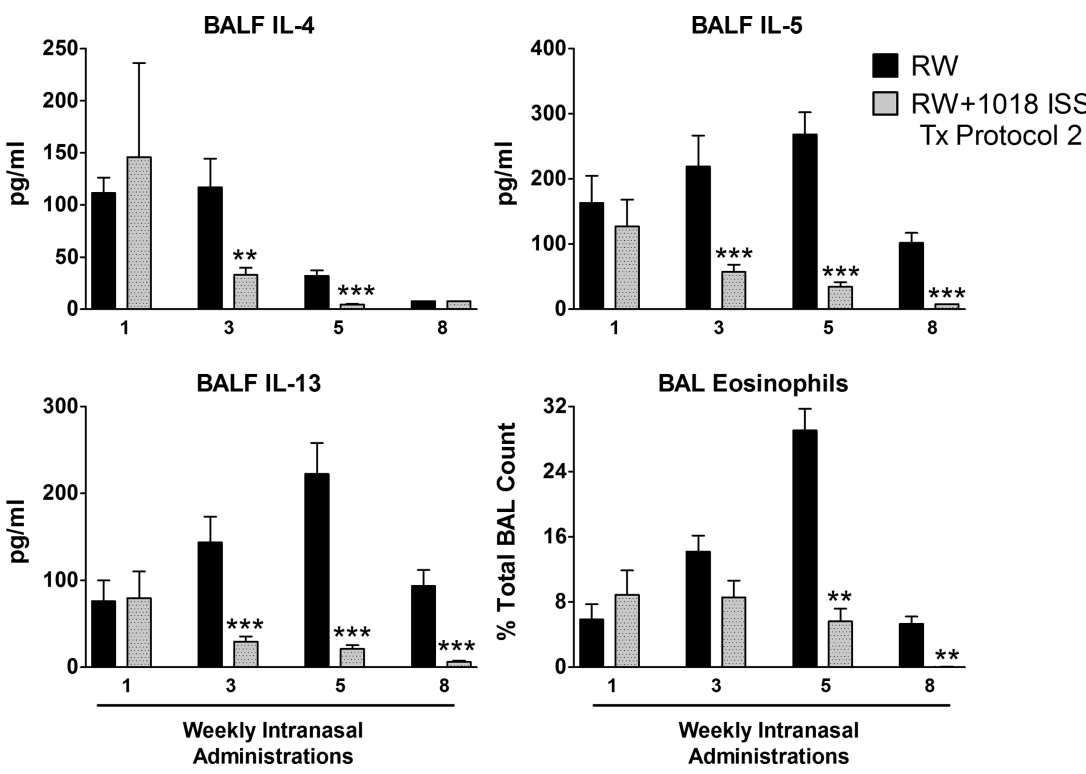

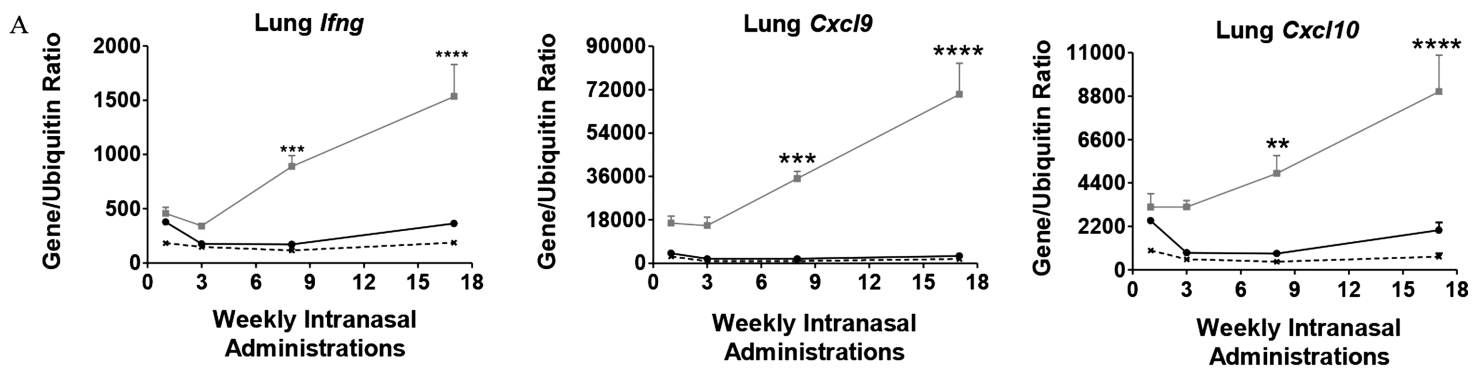

B
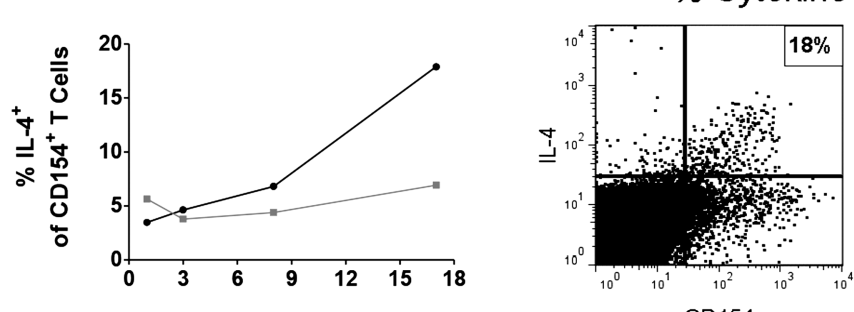

\% Cytokine of CD154+Cells
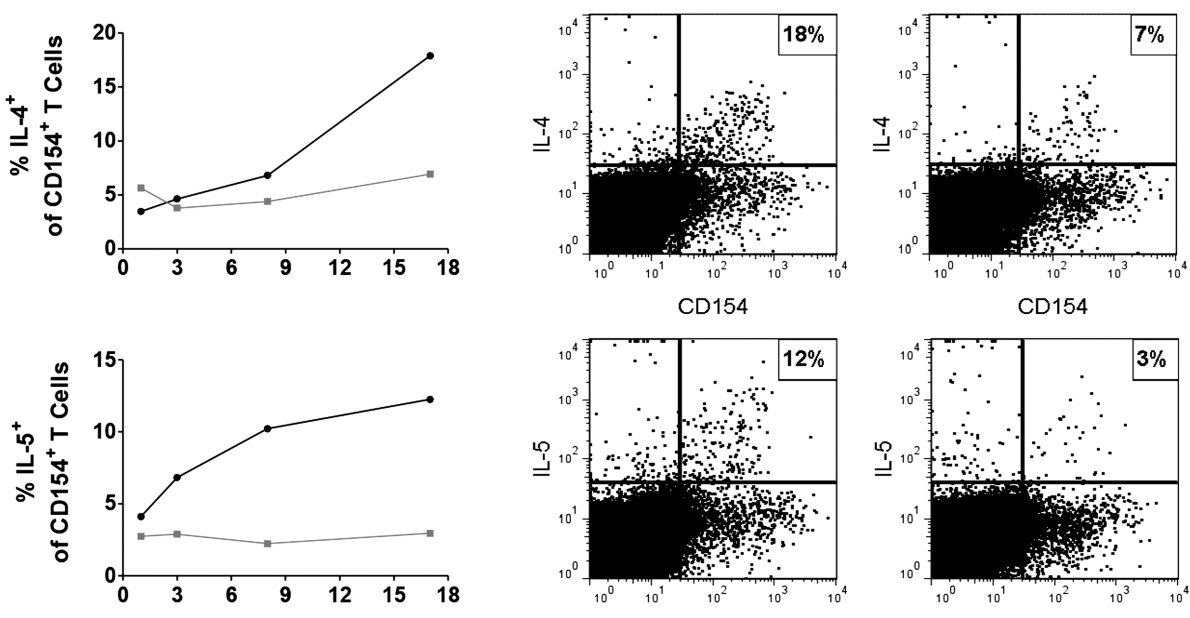

CD154
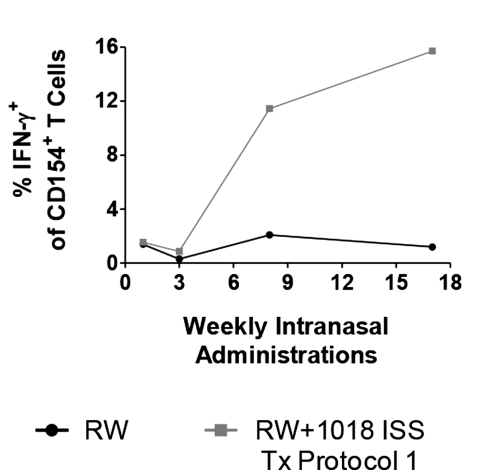

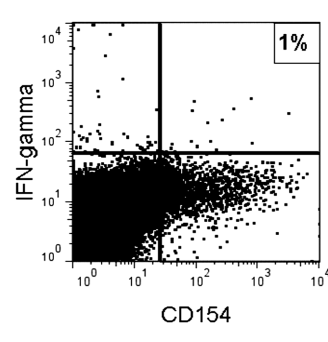

RW

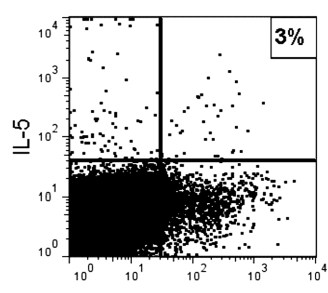

CD154

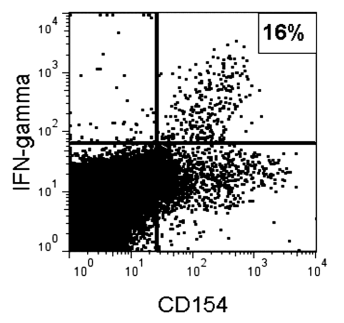

RW + 1018 ISS

Tx Protocol 1

Figure 41018 ISS progressively induces a T helper 1 shift in lungs of treated mice. (A) Lung gene expression is shown as gene/ubiquitin ratio for mice administered Tx protocol 1 for the indicated number of weeks (mean \pm SEM, 5 mice/group), followed by rest and final ragweed (RW) challenge. ${ }^{* *} p<0.01,{ }^{* *} p<0.001,{ }^{* * * *} p<0.0001$ compared with the RW only exposed group. (B) Kinetics of intracellular cytokine expression following ex vivo RW re-stimulation of lung-derived CD4 T cells (pooled/treatment group). Antigen-specific T cells from mice administered Tx protocol 1 for indicated weeks were identified by de novo CD154 expression. Antigen-specific intracellular cytokine expression after 17 weeks of Tx Protocol 1 is also depicted in dot plots. Data are representative of two to four independent experiments. IL, interleukin.

Th1-associated genes. RW +1018 ISS treatments progressively increased expression of Th1-associated genes, Ifng, Cxcl9 and Cxcl10 compared with expression in RW only exposed mice (figure 4A; Tx protocol 1). This Th2-like to Th1-like shift in $\mathrm{RW}+1018$ ISS-treated mice was observed directly in RW-specific lung $\mathrm{T}$ cells (figure 4B). Following ex vivo re-stimulation with RW, lung-derived antigen-specific CD4 $\mathrm{T}$ cells were identified by de novo expression of CD154. ${ }^{21} 22$ After 17 weekly RW only exposures, cells were predominantly Th2-like, but predominantly Th1-like in mice treated for 17 weeks with RW+1018 ISS (Tx protocol 1; figure 4B). Secreted BALF IFN- $\gamma$ protein was below the limit of detection $(<13 \mathrm{pg} / \mathrm{mL})$, suggesting moderate induction of Th1 responses in 1018 ISS treated mice. Nevertheless, the lung Th1 shift was reflected systemically as indicated by reduced $\operatorname{IgE}$ and elevated IgG2a in serum of mice administered RW +1018 ISS (Tx protocol 1 ; see online supplementary figure S2).

To determine the role of IFN $\gamma$ in maintenance of the Th2 to Th1 shift induced by 1018 ISS, mice were administered 13 weekly RW+1018 ISS treatments (Tx protocol 2) and then treated with anti-IFN $\gamma$ antibody, isotype or no antibody during the 4 weekly post-treatment RW exposures. Once established, lasting Th2 suppression was not reversed by IFN $\gamma$ neutralisation. BALF IL-4, IL-5 and serum IgE, and IL-4 or IL-5 expressing $\mathrm{RW}$-specific $\mathrm{T}$ cells in the lung or blood remained low in anti-IFN $\gamma$ treated mice (figure 5A,B). Inhibition of lung $\mathrm{Cxcl} 9$ 

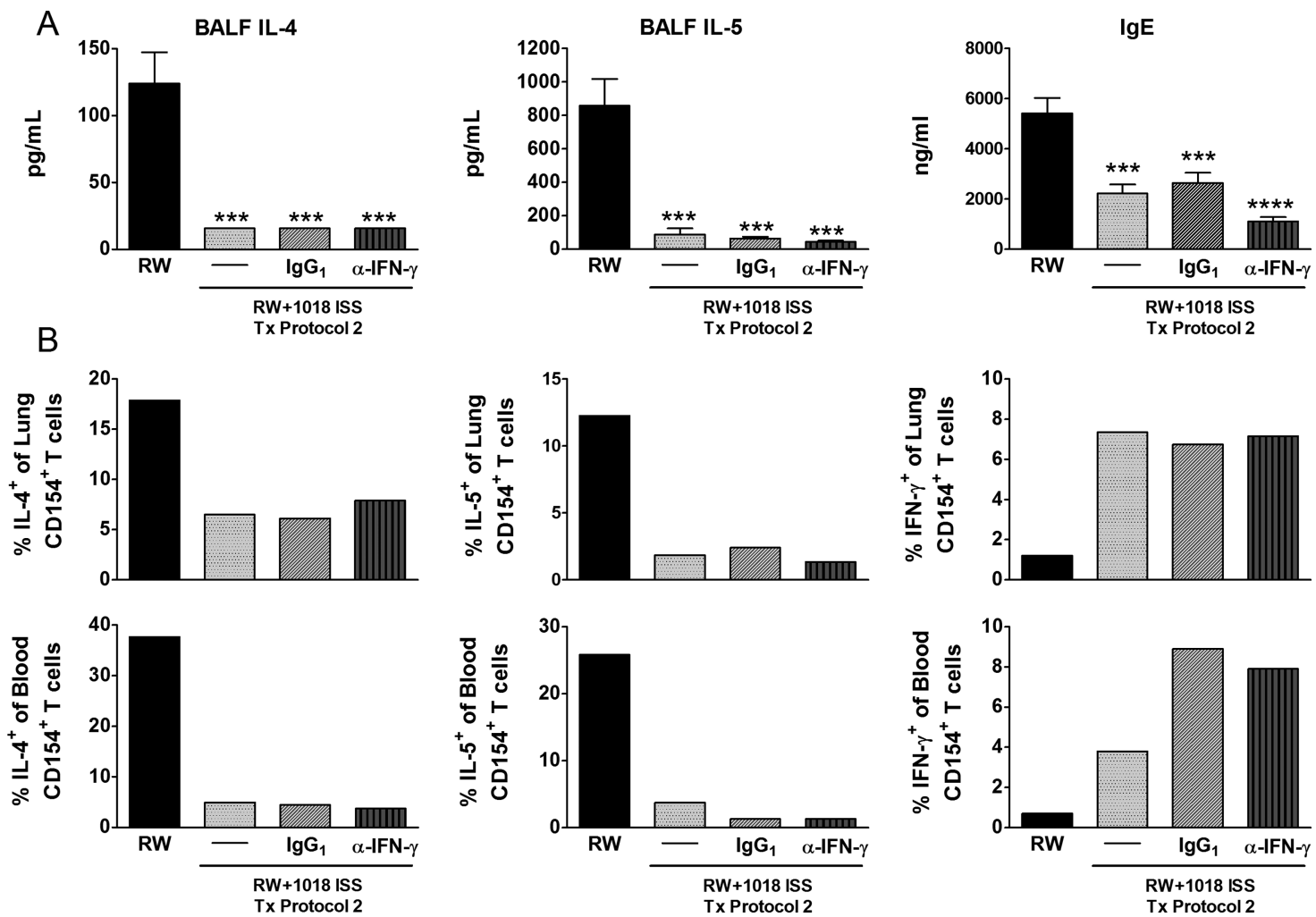

Figure 5 Interferon $\gamma($ IFN $\gamma)$ is not required for maintenance of 1018 ISS-induced disease modification. Mice were administered Tx protocol 2 with 13 weekly ragweed (RW)+1018 ISS treatments, followed by 4 weekly RW exposures, rest and final RW challenge. Neutralising anti-IFN $\gamma$ antibody or isotype control was administered via the intraperitoneal route concurrently with the last 4 weekly RW exposures. (A) Bronchoalveolar lavage fluid (BALF) cytokines and serum IgE (mean \pm SEM, 5 mice/group). ${ }^{* * *} p<0.001,{ }^{* * *} p<0.0001$ compared with the RW only exposed group. (B) Antigen-specific intracellular cytokine expression (tissue pooled/group). Data are representative of two independent experiments. IL, interleukin.

and Cxcl10 expression in RW +1018 ISS-treated mice confirmed the activity of IFN $\gamma$ antibodies (data not shown).

\section{RW-pulsed dendritic cells cannot restore allergic responses in 1018 ISS-treated mice}

A single CpG-ODN treatment inhibits the ability of lung antigen-presenting cells (APCs) to activate Th2 cells. ${ }^{19}$ To determine whether APC dysfunction could explain 1018 ISS-induced durable suppression, mice were challenged with exogenous APC pulsed with RW. Intratracheal challenge with allergen-pulsed BMDCs is comparable to airway allergen challenge and sufficient to induce all features of allergic airway inflammation in sensitised mice. ${ }^{23}$ Here, mice given 5 weekly RW +1018 ISS treatments, followed by 4 weekly RW exposures (Tx protocol 2 ) were challenged with RW-pulsed BMDCs (RWpDCs), untreated DC, RW or saline. RW-exposed mice challenged with RWpDCs had elevated BALF IL-5, IL-13 and eosinophilia, and lung Th2-associated genes (figure 6A). However, RWpDCs did not 'restore' Th2 responses in 1018 ISS-treated mice (figure 6A), but rather induced expression of Th1-associated genes Ifng, Cxcl9 and Cxcl10 (figure 6B). Thus, allergen presented by DCs never exposed to 1018 ISS did not reinstate the Th2 response to RW in 1018-treated mice, indicating that lung APC function defects are not sufficient to explain 1018 ISS-mediated, longlived inhibition of Th2 responses.

\section{ISS induces elevated regulatory T-cell-associated} genes and proteins and increased regulatory T-cell activity Since neither IFN $\gamma$ nor chronic APC suppression could fully account for persistence of 1018 ISS-induced Th2 inhibition, we used microarray analysis for a comprehensive and unbiased approach to identify differences between RW, RW +1018 ISS-treated and control mice. To assess steady-state gene expression, no final RW challenge was administered to mice rested 2 weeks after 16 weekly $\mathrm{RW}+1018$ ISS treatments ( $\mathrm{Tx}$ protocol 1 ). Microarray revealed a pattern of 1018 ISS-induced upregulation of regulatory T-cell $\left(\mathrm{T}_{\mathrm{Reg}}\right)$-associated genes. Genes for Foxp3, IL-10 and transforming growth factor $\beta$ (TGF $\beta$ ) (Tgfb1), and

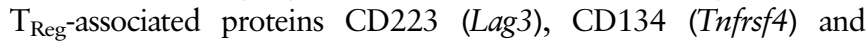
CD25 (Il2ra) were all expressed at significantly higher levels in 1018 ISS-treated mice than RW-exposed or control mice (figure 7A). The expected Th2 or CpG-ODN-inducible gene expression was also evident in RW and RW+1018 ISS treated mice, respectively (see online supplementary tables S1 and S2).

Elevated $\mathrm{T}_{\mathrm{Reg}}$-associated gene expression in $\mathrm{RW}+1018$ ISS-treated mice was reflected at the protein level, except for $\mathrm{CD} 25$, as shown by flow cytometry of lung $\mathrm{CD} 3{ }^{+} \mathrm{CD} 4^{+}$T cells (pooled/treatment group) from $\mathrm{RW}+1018$ ISS-treated compared with RW-exposed mice (figure 7B; Tx protocol 2, 5 weekly RW +1018 ISS, followed by 4 weekly RW, no final challenge). Additionally, $\mathrm{CD} 3{ }^{+} \mathrm{CD} 4{ }^{+} \mathrm{Foxp} 3^{+} \mathrm{T}$ cells from lungs of 1018 ISS-treated mice (Tx protocol 1, 16 weekly $\mathrm{RW}+1018$ ISS, no final challenge) demonstrated an increased proportion of $\mathrm{CD} 25^{+} \mathrm{CD} 127^{\text {lo }}$ T cells containing increased $\mathrm{CD} 39^{+} \mathrm{CD} 73^{+}$ $\mathrm{T}$ cells (figure $7 \mathrm{C}$ ). $\mathrm{CD} 39^{+} \mathrm{CD} 73^{+}$expression ranged from $\sim 5-$ $8 \%$ for T cells from RW +1018 ISS-treated mice to $\sim 0-2 \%$ for $\mathrm{T}$ cells from RW-exposed mice. Lastly, lung $\mathrm{CD} 4{ }^{+} \mathrm{CD} 25^{+}$'regulatory' $\mathrm{T}$ cells were incubated at different ratios with $\mathrm{CD} 4{ }^{+} \mathrm{CD} 25^{-}$'effector' $\mathrm{T}$ cells $\left(\mathrm{T}_{\mathrm{Eff}}\right)$ from the lungs of RW-exposed mice as well as irradiated spleen cells (as APCs). 
A) Th2 Responses

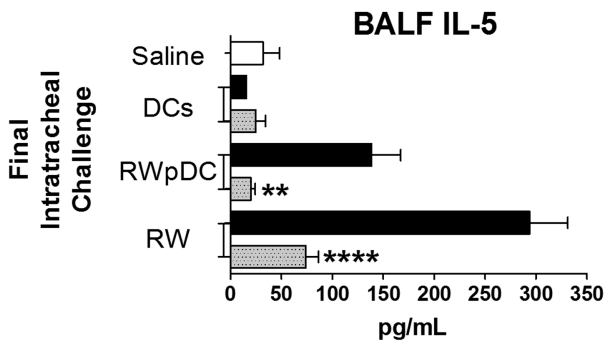

Lung Fizz1

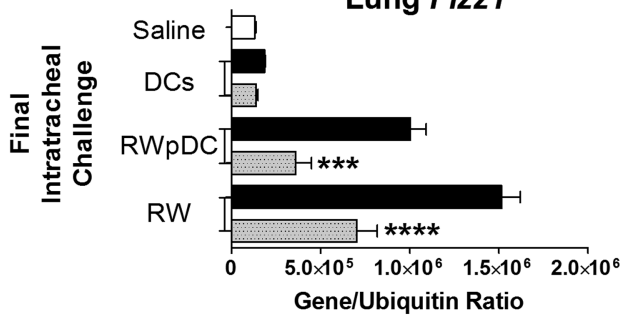

B) Th1 Responses

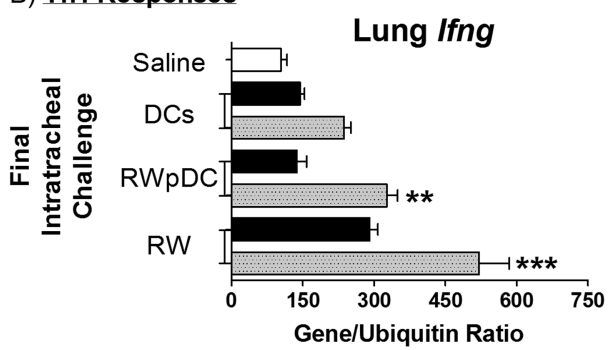

Weekly Intranasal Exposures:

Saline RW RW+1018 ISS Tx Protocol 2

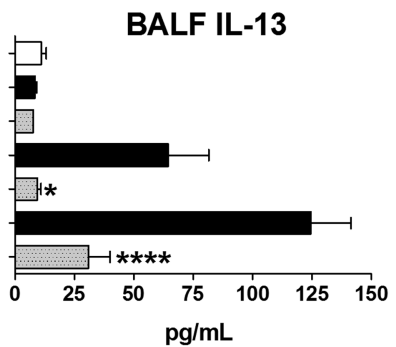

Lung II13
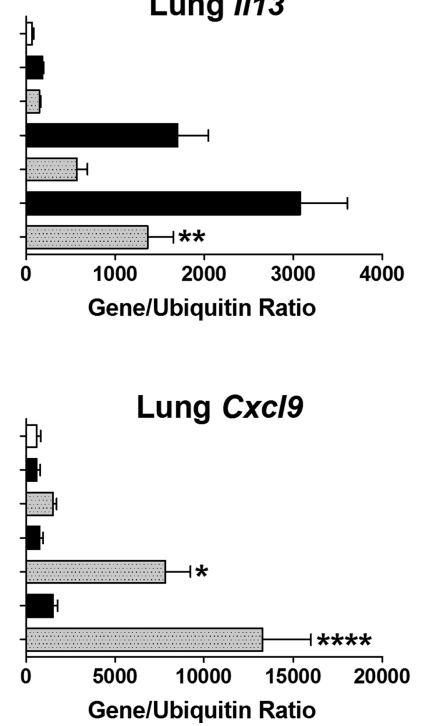

BAL Eosinophils

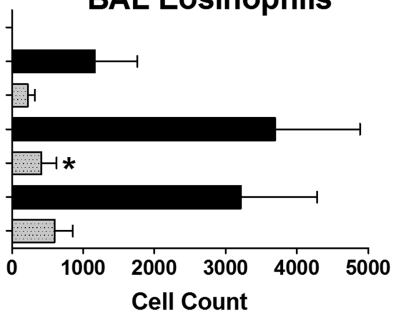

Lung Ccl11

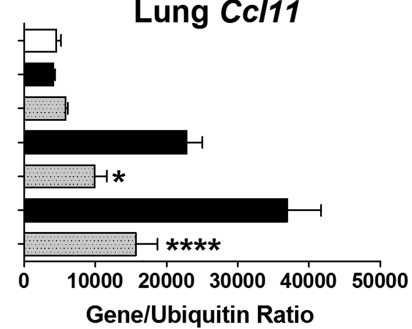

Lung Cxc/10

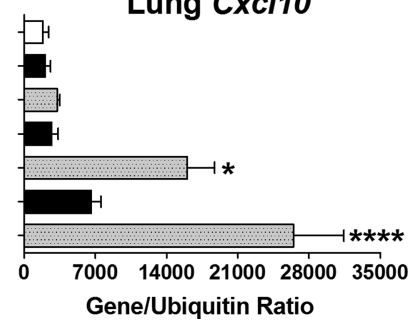

Figure 6 Ragweed (RW)-pulsed marrow-derived dendritic cells (BMDCs) do not restore T helper 2 (Th2) responses in 1018 ISS-treated mice. Mice were given Tx protocol 2: 5 weekly RW+1018 ISS treatments or RW only, followed by 4 weekly RW exposures, 2 weeks of rest and a final challenge of pulsed DCs (RWpDCs), DCs, RW or saline administered intratracheally. (A) Th2 cytokines, eosinophils and gene expression, and (B) Th1 gene expression are shown for the different treatment groups (mean \pm SEM, 8 mice/group). ${ }^{*} p<0.05,{ }^{* *} p<0.01,{ }^{* * *} p<0.001,{ }^{* * * *} p<0.0001$ compared with the corresponding RW only weekly exposures group. Data are representative of two independent experiments. IL, interleukin.

On a per cell basis, $\mathrm{T}_{\text {Reg }}$ cells from RW +1018 ISS-treated mice suppressed anti-CD3 stimulated $\mathrm{T}_{\text {Eff }}$ cell proliferation more effectively than $\mathrm{T}_{\text {Reg }}$ from RW-exposed mice (figure 7D). These data demonstrate that a short course of 1018 ISS treatment induces a functional regulatory T-cell response associated with reduced allergic lung responses in this chronic asthma model.

\section{DISCUSSION}

Defining the conditions for generating durable suppression of allergic airway disease is a key step in developing a CpG-ODN treatment regimen for humans. Previous reports of CpG-ODN treatment in longer-term murine asthma models (3-6 months) examined effects within days of the last dose administered or only after a very limited number of allergen exposures following therapy, a scenario not representative of natural chronic allergen exposure. ${ }^{11-13}$ To model human allergic asthma as closely as possible, we developed a long-term asthma model using weekly exposures to a clinically relevant allergen (RW) administered at a dose $(0.5 \mu \mathrm{g})$ much lower than previously reported in limited RW allergen exposure studies. ${ }^{24} 25$ Lung Th2 inflammation was evident in RW and RW +1018 ISS exposed groups after the first intranasal exposure/treatment and was sustained for half a year in mice receiving weekly RW-only exposures. Using this model, we tested whether a limited course of inhaled CpG-ODN therapy would suppress allergic lung inflammation and whether this effect would be durable in the presence of weekly allergen exposures continuing after treatment cessation. The drug was delivered directly to the lung by intranasal administration, which has been shown to be more effective than distal delivery $^{26}$ and is the intended route for clinical application.

Our data show that 12 weekly 1018 ISS treatments induce suppression of allergic airway inflammation lasting through 13 subsequent weekly RW exposures and a final high-dose $(5 \mu \mathrm{g})$ RW allergen challenge 2 weeks later (ie, suppression was maintained for a total of 15 weeks post treatment). Therefore the period of post-treatment disease inhibition lasted longer than the treatment period and likely may last even longer, as no loss of efficacy was observed. A minimum of 5 weekly 1018 ISS doses were required to suppress established Th2 responses and the suppression lasted for at least 6 weeks without loss of efficacy. Thus, CpG-ODN offers the potential to induce diseasemodifying effects in allergic asthma after a relatively brief treatment regimen, possibly to multiple allergen sensitivities. In comparison, allergen-specific immunotherapy generally requires much longer treatment regimens (2-3 years) to induce sustained mono-specific remission lasting in time up to the length of the treatment regimen. ${ }^{27}$

CpG-ODN are known to induce Th1 responses ${ }^{6} 28$ and the durable suppression of Th2 responses achieved here was accompanied by a moderate Th1-like shift in the lungs of 1018 
A
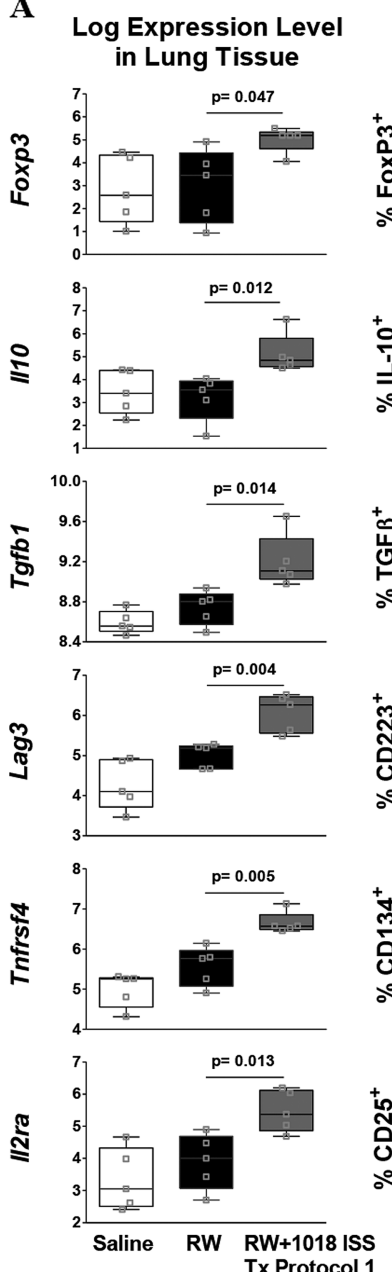

B
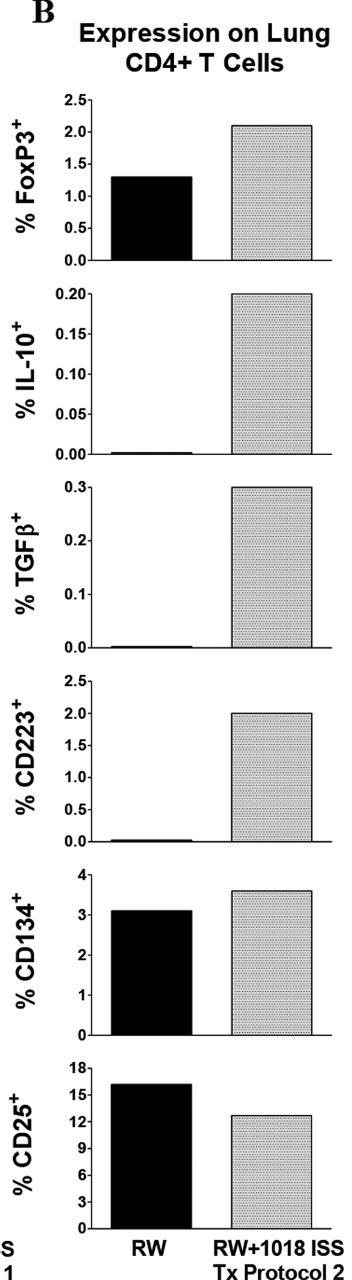

C

RW
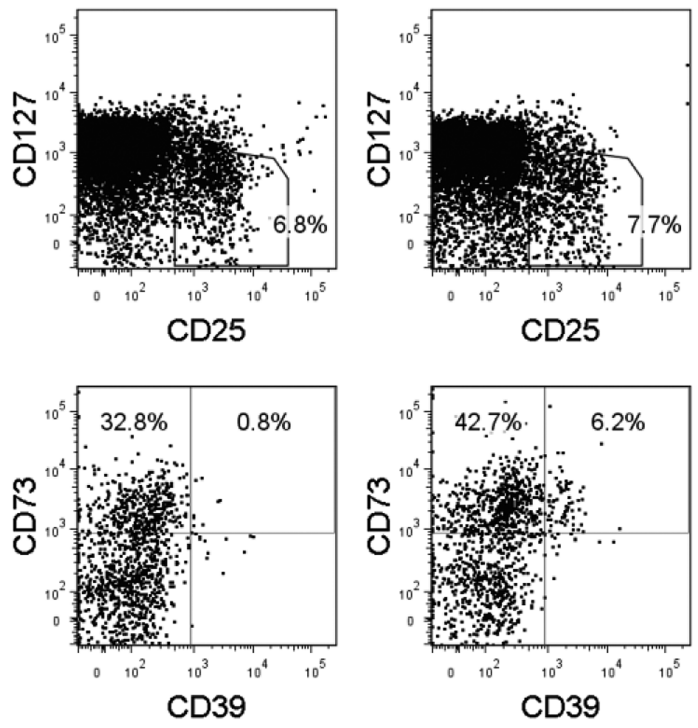

D

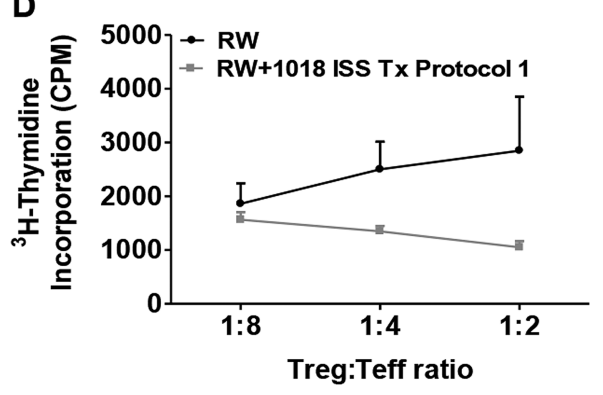

Figure 71018 ISS-induced disease modification is associated with induction of a lung $T_{\text {Reg }}$ response. (A) Lung tissue expression of $T_{\text {Reg }}$-associated genes revealed by microarray analysis ( 16 weeks of Tx protocol 1 , no final challenge). Student's $t$ test $p$ value, compared with ragweed (RW), is indicated on box-and-whisker plots (1 experiment/n=5/group). (B) Expression of $\mathrm{T}_{\text {Reg }}$ markers on lung CD4 T cells and ex vivo anti-CD3 (10 $\mu \mathrm{g} / \mathrm{mL}$ ) +anti-CD28 $(2 \mu \mathrm{g} / \mathrm{mL})$ stimulated intracellular cytokines by flow cytometry (pooled/treatment group; 5 weeks of Tx protocol 2, then 4 weeks of RW only, no final challenge). (C) CD39 and CD73 expression on lung CD25 hi CD127 ${ }^{\text {lo }} \mathrm{CD} 4^{+} \mathrm{CD} 25^{+}$Foxp3 ${ }^{+} \mathrm{T}$ cells (pooled/treatment group; same protocol as A). (D) Proliferative responses of anti-CD3-stimulated lung $T_{E f f}\left(C D 4^{+} C D 25^{-}\right)$from RW-exposed mice incubated with irradiated splenic antigen-presenting cells (APCS) and lung $\mathrm{T}_{\text {Reg }}\left(\mathrm{CD} 4^{+} \mathrm{CD} 25^{+}\right)$from RW or RW+1018 ISS treated mice at indicated ratios (mean \pm SEM of triplicate cultures, cells pooled/treatment group; 7 weeks of Tx protocol 1, no final challenge). Average for $\mathrm{T}_{\text {Eff }}$ in absence of $\mathrm{T}_{\text {Reg }}$ was 2000 CPM. Data are representative of three to five independent experiments.

ISS-treated mice. The continuing presence of a small population of lung RW-specific Th2 cells in 1018 ISS-treated mice suggests that the treatment regimen may not completely eliminate Th2 cells, but substantially compromises their function. In vivo IFN $\gamma$ neutralisation after completion of 1018 ISS treatment did not reverse lung Th2 suppression (figure 5), showing that the Th1 shift is not required for continued Th2 inhibition, although it may still contribute. Sustained impairment of lung DCs cannot account for 1018 ISS-induced long-term suppression of Th2 responses as delivery of fresh antigen-loaded DCs directly to the lungs did not reignite local Th2 inflammation in 1018 ISS-treated mice (figure 6).

Animal model and clinical studies suggest that regulatory $\mathrm{T}$ cells have the potential to contribute to suppression of allergic airway disease. Adoptive transfer of $\mathrm{CD} 4^{+} \mathrm{CD} 25^{+} \mathrm{T}_{\mathrm{Reg}}$ cells into sensitised mice before or after allergen challenge reduces allergic airway inflammation ${ }^{29} 30$ and, conversely, $\mathrm{T}_{\text {Reg }}$ depletion exacerbates allergic responses. ${ }^{31}$ In patients on specific immunotherapy, induction of $\mathrm{CD} 4{ }^{+} \mathrm{CD} 25^{+}$Foxp $3^{+} \mathrm{T}$ cells and IL-10 expression has been correlated with $\mathrm{IgG} 4 / \mathrm{IgE}$ ratios and clinical efficacy for suppression of seasonal allergies. ${ }^{32}{ }^{33}$ Indeed, analysis of allergen-specific T cells in healthy and allergic individuals suggests that the fine balance between $T_{\text {Reg }}$ and Th2 cells may determine the development of allergic disease. ${ }^{34}$ In the present study, expression of multiple $\mathrm{T}_{\mathrm{Reg}}$-associated genes was higher in lungs of 1018 ISS-treated mice (figure 7) and this was reflected at the protein level, except for CD25, which is also expressed on activated T cells, likely still present in the lungs at this point. ${ }^{35}$ Functionally, increased $\mathrm{T}_{\text {Reg }}$ activity in 1018 ISS-treated mice was confirmed in an ex vivo assay. We have not defined the precise $T_{\text {Reg }}$ mechanism(s) operating in our system, but given the upregulation of multiple $T_{\text {Reg }}$ markers in the lungs, several mechanisms may be involved. The mixed $\mathrm{Th} 1 / \mathrm{T}_{\mathrm{reg}}$ response that resulted from 1018 ISS treatments suggests the possibility that Th1-like regulatory cells expressing IFN $\gamma$ and IL-10 may form a component of this response. However, IFN $\gamma$ expression by RW-specific lung T cells increased with successive 1018 ISS doses (figure 4), whereas IL-10 expression by these 
cells did not differ between treatment groups at any time point (data not shown), indicating predominant induction of RW-specific Th1 cells. We did not, however, co-stain for antigen-specific T cells expressing IL-10 and IFN $\gamma$. Nevertheless, a role for IL-10 is possible as ex vivo polyclonal re-stimulation of the entire lung T-cell population revealed slightly elevated IL- $10^{+} \mathrm{CD}^{+} \mathrm{T}$ cells from 1018 ISS-treated mice and lung IL-10 gene expression was also elevated in 1018 ISS-treated mice (figure 7). In this readout, other cell populations including bystander T cells may be contributing to the IL-10 response.

In summary, we evaluated whether repeated treatments with a TLR9 ligand, administered directly into the mouse respiratory tract, lead to a disease-modifying alteration of allergic airway inflammation caused by a common human allergen, RW pollen. Durable inhibition of the response to allergen challenge was achieved in as few as 5 weekly treatments with 1018 ISS coadministered with allergen and was associated with a shift from a Th2 to a mixed Th1/ $\mathrm{T}_{\text {Reg }}$ response. Neither IFN $\gamma$ nor long-lived inhibition of lung APC function was required for disease modification, although both mechanisms may contribute to the overall effect. These findings support development of inhaled CpG-ODN as a novel disease-modifying therapy for allergic asthma.

Acknowledgements We gratefully thank Drs Cristiana Guiducci, Franck Barrat, Holger Kanzler and Yasmin Belkaid for their helpful advice and discussions. We also thank Robert Milley for preparation of 1018 ISS and ragweed; Henry Lopez, Steve Noonan and Shannon Smart for assistance with in vivo treatment procedures; Hector Nolla for flow cytometry sorting; and Carol Culwell for administrative assistance.

Contributors JDC conducted experiments, analysed data, prepared figures, advised on study design, and wrote and submitted the final manuscript approved by all authors. SAK conducted the majority of the experiments, analysed data, prepared figures and contributed to manuscript writing. HMK, JAL, RS, MC and CRC performed some of the experimental work. HS analysed microarray data. CML advised on study design. RLC advised on study design and edited the article. EMH designed the study and supervised the project.

Funding This work was funded by Dynavax Technologies Corporation.

Competing interests JDC, SAK, HMK, JAL, RS, MC, CRC, RLC and EMH are or were employees of Dynavax Technologies Corporation which funded the work.

Provenance and peer review Not commissioned; externally peer reviewed.

\section{REFERENCES}

1 Fanta CH. Asthma. N Engl J Med 2009;360:1002-14.

2 Frew AJ. Injection immunotherapy. British Society for Allergy and Clinical Immunology Working Party. BMJ 1993;307:919-23.

3 Bousquet J, Lockey R, Malling HJ. Allergen immunotherapy: therapeutic vaccines for allergic diseases. A WHO position paper. J Allergy Clin Immunol 1998;102(4 Pt 1):558-62.

4 Jacobsen L, Valovirta E. How strong is the evidence that immunotherapy in children prevents the progression of allergy and asthma? Curr Opin Allergy Clin Immunol 2007:7:556-60.

5 Akdis CA. Therapies for allergic inflammation: refining strategies to induce tolerance. Nat Med 2012;18:736-49.

6 Krieg AM. CpG motifs in bacterial DNA and their immune effects. Annu Rev Immunol 2002:20:709-60.

7 Broide DH, Stachnick G, Castaneda D, et al. Systemic administration of immunostimulatory DNA sequences mediates reversible inhibition of Th2 responses in a mouse model of asthma. J Clin Immunol 2001;21:175-82.

8 Ikeda RK, Nayar J, Cho JY, et al. Resolution of airway inflammation following ovalbumin inhalation: comparison of ISS DNA and corticosteroids. Am J Respir Cell Mol Biol 2003;28:655-63.

9 Fonseca DM, Paula MO, Wowk PF, et al. IFN-gamma-mediated efficacy of allergen-free immunotherapy using mycobacterial antigens and CpG-ODN. Immuno Cell Biol 2011:89:777-85.

10 Jain VV, Kitagaki K, Businga T, et al. CpG-oligodeoxynucleotides inhibit airway remodeling in a murine model of chronic asthma. J Allergy Clin Immunol 2002;110:867-72.
11 Youn CJ, Miller M, Baek KJ, et al. Immunostimulatory DNA reverses established allergen-induced airway remodeling. J Immunol 2004;173:7556-64.

12 Cho JY, Miller M, McElwain K, et al. Remodeling associated expression of matrix metalloproteinase 9 but not tissue inhibitor of metalloproteinase 1 in airway epithelium: modulation by immunostimulatory DNA. J Allergy Clin Immunol 2006;117:618-25.

13 Kline JN, Kitagaki K, Businga TR, et al. Treatment of established asthma in a murine model using CpG oligodeoxynucleotides. Am J Physiol Lung Cell Mol Physiol 2002;283:L170-9.

14 Fanucchi MV, Schelegle ES, Baker GL, et al. Immunostimulatory oligonucleotides attenuate airways remodeling in allergic monkeys. Am J Respir Crit Care Med 2004:170:1153-7.

15 Tulic MK, Fiset PO, Christodoulopoulos P, et al. Amb a 1-immunostimulatory oligodeoxynucleotide conjugate immunotherapy decreases the nasal inflammatory response. J Allergy Clin Immunol 2004;113:235-41.

16 Simons FE, Shikishima $Y$, Van Nest G, et al. Selective immune redirection in humans with ragweed allergy by injecting Amb a 1 linked to immunostimulatory DNA J Allergy Clin Immunol 2004;113:1144-51.

17 Creticos PS, Schroeder JT, Hamilton RG, et al. Immunotherapy with a ragweed-toll-like receptor 9 agonist vaccine for allergic rhinitis. $N$ Eng/ J Med 2006:355:1445-55

18 Marshall JD, Fearon K, Abbate C, et al. Identification of a novel CpG DNA class and motif that optimally stimulate B cell and plasmacytoid dendritic cell functions. J Leukoc Biol 2003;73:781-92

19 Hessel EM, Chu M, Lizcano J0, et al. Immunostimulatory oligonucleotides block allergic airway inflammation by inhibiting Th2 cell activation and IgE-mediated cytokine induction. J Exp Med 2005;202:1563-73.

20 Lutz MB, Kukutsch N, Ogilvie AL, et al. An advanced culture method for generating large quantities of highly pure dendritic cells from mouse bone marrow. J Immunol Methods 1999:223:77-92.

21 Campbell JD, Buchmann P, Kesting S, et al. Allergen-specific T cell responses to immunotherapy monitored by CD154 and intracellular cytokine expression. Clin Exp Allergy 2010;40:1025-35.

22 Frentsch $M$, Arbach 0 , Kirchhoff $D$, et al. Direct access to CD4+ T cells specific for defined antigens according to CD154 expression. Nat Med 2005; 11:1118-24

23 van Rijt LS, Jung $S$, Kleinjan A, et al. In vivo depletion of lung CD11c+ dendritic cells during allergen challenge abrogates the characteristic features of asthma. J Exp Med 2005:201:981-91.

24 Sur S, Wild JS, Choudhury BK, et al. Long term prevention of allergic lung inflammation in a mouse model of asthma by $\mathrm{CpG}$ oligodeoxynucleotides. J Immunol 1999;162:6284-93.

25 Yadav UC, Aguilera-Aguirre L, Boldogh I, et al. Aldose reductase deficiency in mice protects from ragweed pollen extract (RWE)-induced allergic asthma. Respir Res 2011;12:145.

26 Takabayashi K, Libet L, Chisholm D, et al. Intranasal immunotherapy is more effective than intradermal immunotherapy for the induction of airway allergen tolerance in Th2-sensitized mice. J Immunol 2003;170:3898-905.

27 Stelmach I, Sobocinska A, Majak P, et al. Comparison of the long-term efficacy of 3- and 5-year house dust mite allergen immunotherapy. Ann Allergy Asthma Immuno 2012;109:274-8.

28 Roman M, Martin-Orozco E, Goodman JS, et al. Immunostimulatory DNA sequences function as T helper-1-promoting adjuvants. Nat Med 1997;3:849-54.

29 Kearley J, Barker JE, Robinson DS, et al. Resolution of airway inflammation and hyperreactivity after in vivo transfer of CD4+CD25+ regulatory T cells is interleukin 10 dependent. J Exp Med 2005:202:1539-47.

30 McGee HS, Agrawal DK. Naturally occurring and inducible T-regulatory cells modulating immune response in allergic asthma. Am J Respir Crit Care Med 2009:180:211-25.

31 Lewkowich IP, Herman NS, Schleifer KW, et al. CD4+CD25+ T cells protect against experimentally induced asthma and alter pulmonary dendritic cell phenotype and function. J Exp Med 2005;202:1549-61

32 Bohle B, Kinaciyan T, Gerstmayr $M$, et al. Sublingual immunotherapy induces IL-10-producing T regulatory cells, allergen-specific T-cell tolerance, and immune deviation. J Allergy Clin Immunol 2007;120:707-13.

33 Radulovic $S$, Jacobson MR, Durham SR, et al. Grass pollen immunotherapy induces Foxp3-expressing CD4+ CD25+ cells in the nasal mucosa. J Allergy Clin Immunol 2008; 121:1467-72, 72 e1.

34 Akdis M, Verhagen J, Taylor A, et al. Immune responses in healthy and allergic individuals are characterized by a fine balance between allergen-specific $T$ regulatory 1 and T helper 2 cells. J Exp Med 2004;199:1567-75.

35 Julia V, Hessel EM, Malherbe L, et al. A restricted subset of dendritic cells captures airborne antigens and remains able to activate specific $T$ cells long after antigen exposure. Immunity 2002;16:271-83. 